I. Introduction ....................................................................................... 21

II. Tableau des cours........................................................................................... 2

Les grands continents du globe du droit international privé ............................ 28

L’évolution par Etat ou par groupes d'Etats, ou dans
un cadre fédéralou quasi fédéral ................................................................ 30

L'évolution dans le temps et les rapports avec le droit internationalpublic ....... 33

Le débat atlantique .............................................................................. 34

Les cours généraux ................................................................................ 35

La procédure internationale de droit privé ..................................................... 37

D'autres thèmes .......................................................................................... 39

La coopération internationale en vue de la mise au point de règles communes 41

III. L'autonomie des parties à un contrat et les lois de police ............................... 43

Les lois de police ............................................................................... 44

L'autonomie des parties à un contrat international, dite l'autonomie de la volonté

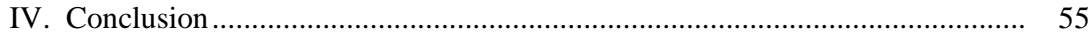

\title{
BLANCHE
}

\section{NOTICE BIOGRAPHIQUE}

Antoon (Teun) Victor Marie Struycken, né en 1936 à Breda-Ginneken, Pays-Bas.

Etudes à l'Université catholique de Nimègue, Pays-Bas (1954-1959), au Bologna

Center of the Johns Hopkins School for Advanced International Studies (1961-1962),

à la faculté de droit et des sciences économiques de l'Université de Paris (1962-1963).

Assistant à la faculté de droit de l’Université catholique de Nimègue (1963-1971).

Docteur en droit, Nimègue (1970).

Professeur de droit international privé, de droit civil et de droit privé comparé à la faculté de Nimègue (depuis 1971).

Président de la section Droit du Conseil académique national (1974-1980). Membre du conseil d'administration de l'Internationaal Juridisch Instituut (IJI), La Haye (depuis 1976) ; président depuis 1995.

Doyen de la faculté de droit de Nimègue (1987-1991), doyen honoraire.

Juge suppléant au tribunal d'arrondissement d'Arnhem (depuis 1972) ; con-seiller suppléant à la cour d'appel d'Arnhem (depuis 1978).

Membre de la Commission permanente néerlandaise pour les questions de l'état civil et de la section néerlandaise de la Commission internationale de l'état civil (CIEC) (1965-1985).

Membre (depuis 1975), et président depuis 1995, de la Commission d'Etat néerlandaise de droit international privé.

Délégué des Pays-Bas aux seizième, dix-septième et dix-huitième sessions de la Conférence de La Haye de droit international privé ; président de la dix-huitième session.

Membre du Curatorium de l'Académie de droit international de La Haye (depuis 1994).

Note sur l'arrêt du Hoge Raad du 13 mai 1966 (Alnati), Revue critique de droit international privé, 1967, p. 522.

« De gracieuze jurisdictie in het internationaal privaatrecht — beschouwingen over de rechtsmacht van de Nederlandse rechter », thèse, Nimègue, 1970, Deventer, 1970.

" Naar eenheid van rechtsbedeling in Europa » (sur la Convention de Bruxelles de 1968), leçon inaugurale, 1971, Deventer, 1971.

« Kan een rechtspersoon naar Nederlands recht ook worden opgericht ten over--staan van een vreemde notaris ?», De Naamlooze Vennootschap, 1973, pp. 165-175.

«Vertegenwoordiging krachtens volmacht in het Nederlands internationaal privaatrecht », WPNR (1976), pp. 5346-5347.

" De rechterlijke bevoegdheid volgens het EEG Jurisdictie- en Executieverdrag ", rapport pour l'Association néerlandaise de droit international, 1978, série de l'Association $\mathrm{n}^{\circ}$ 77. (Résumé en anglais dans Netherlands International Law Review, 1978, pp. 354-363.) 
«Het Europees Executieverdrag en de woonplaats van de rechtspersoon », De Naamlooze Vennootschap, 1978, pp. 45-51.

« Buitenlandse adopties aan het loket », Het Personeel Statuut, 1980, pp. 67-73, 8286.

« Doorbraak van aansprakelijkheid in het internationaal privaatrecht », WPNR (1981), 5575.

« Artikel 23 BBS, een netelig element van het takenpakket van de Haagse ambtenaar van de burgerlijke stand », Het Personeel Statuut, 1982, pp. 18-27.

«Verenigingen als wereldburger: qua patet orbis », Mélanges W. C. L. van der Grinten (Goed en trouw), Zwolle, 1984, pp. 267-289.

"Pensioenverevening in het internationaal privaatrecht », Mélanges E. E. A. Luij-ten (Een kapitein, twee schepen), 1984, pp. 125-139.

«La lex mercatoria dans le droit des contrats internationaux », recueil des Journées René Savatier (Poitiers, 24-25 octobre 1985), L’évolution contemporaine du droit des contrats, Publications de la faculté de droit et des sciences sociales de Poitiers, t. XV, Paris, 1986, pp. 207-229.

"De ondernemingskamer vanuit IPR-gezichtspunt bekeken ", Mélanges J. M. M. Maeijer (Van vennootschappelijk belang), Zwolle, 1988, pp. 321-336.

"Quelques réflexions sur la juridiction gracieuse en droit international privé ", communication du 15 mai 1987, Travaux du Comité français de droit international privé, années 1986-1987/1987-1988, Paris, 1989, pp. 105-133.

« Acquisition et perte de la nationalité néerlandaise ", dans le recueil du colloque Poitiers-Nimègue, 9-11 mai 1988, La condition juridique de l'étranger, hier et aujourd'hui, faculté de droit de Nimègue, 1988, pp. 3-19.

« Het handelsrecht hoe langer hoe meer over de grenzen heen », article au recueil d'un colloque national sur «Cent cinquante ans du Code de commerce néer-landais » (150 jaar Wetboek van Koophandel), Deventer, 1988, pp. 77-86.

«La Convention de Munich sur la loi applicable aux noms et prénoms », Revista española de Derecho internacional, 1990, p. 153. Texte adapté et traduit en italien dans Rivista di diritto internazionale privato e processuale, 1991, pp. 573-592.

«Les conséquences de l'intégration européenne sur le développement du droit international privé ", Recueil des cours de l'Académie de droit international, tome 232 (1992), pp. 259-383.

"The Hague Convention on the Law Applicable to Succession to the Estates of Deceased Persons 1988, a Civilian Perspective ", Annals 1992 (Part 35), International Academy of Estate and Trust Law.

«L'adoption internationale », dans le Recueil du Premier Congrès européen de la famille (Barcelone, 11-13 mars 1993), pp. 29-41.

«Coördinerende en Tamara-verdragen ", article sur certains types de conventions internationales de droit international privé aux Mélanges J. van Rijn van Alkemade (Grensoverschrijdend privaatrecht), Deventer, 1993, pp. 259-274.

" Van verscheidenheid van recht tot eenheid ; een trap met vele treden », article sur les degrés d'unification et de coordination des systèmes de droit privé, numéro spécial de la revue Rechtsgeleerd Magazijn Themis à l'occasion du centenaire de la Conférence de La Haye de droit international privé, 1993, pp. 237-249.

« Het Haagse Erfrechtverdrag komt eraan ! , article sur la Convention de La Haye sur la succession à cause de mort à la revue WPNR (1993), 6092, pp. 351-356, numéro spécial à l'occasion du centenaire de la Conférence de La Haye.

« La mission de l'arbitre en ce qui concerne la détermination des règles de droit applicables au commerce international », Mélanges D. Kokkini-Iatridou (Comparability and Evaluation), Dordrecht, Martinus Nijhoff, 1994, pp. 369-381.

«Favor matrimonii ou favor divortii ? Point capital d'une politique à long terme dans le domaine de la santé publique mentale ", contribution à un colloque, publiée à la revue FJR (Familie- en jeugdrecht), 1994, pp. 101-106.

«Forum shopping », article au recueil du congrès du 8 juin 1995 de l'Association néerlandaise des avocats du commerce international (NAVIH), 19 pages.

« L'adolescent en droit civil », article aux actes du colloque Nimègue-Poitiers, 1994, Publications de la faculté de droit et des sciences sociales de Poitiers, t. XXVI (1995), Paris, PUF, pp. 115-128.

«Régimes matrimoniaux - banc d'essai de la codification internationale du droit international privé ", Mélanges Georges A.-L. Droz (E Pluribus Unum), La Haye, Martinus Nijhoff, 1996, pp. 445-460.

«Distributie bepaalt attributie », rapport pour un congrès sur la compétence internationale et les règles $\mathrm{y}$ relatives que devrait contenir un nouveau Code de procédure civile, numéro spécial de la revue NIPR, 1996, éd. T. M. C. Asser Instituut, La Haye, pp. 19-43.

« La signification dans l'espace judiciaire européen », dans le recueil La cooperazione giudiziaria nell'Europa dei cittadini, séminaire à Rome organisé par le Ministero di Grazia e Giustizia, Documenti Giustizia-I, 1996, col. 91-106.

«Veelheid van rechtsbronnen, één IPR » (sur quelques problèmes tenant à la pluralité de sources de droit, à résoudre lors de la codification du droit international privé à 
l'échelle nationale), article à la revue néerlandaise Ars Aequi, 1996, numéro spécial, pp. 61-68.

"Le droit religieux et son application par les juridictions civiles et religieuses », Quinzième Congrès international de droit comparé (Bristol, 1998), rapports néerlandais, Anvers, Groningue, 1998, pp. 71-90. 


\section{Introduction}

En cette année 1998, l’Académie de droit international de La Haye célèbre son soixantequinzième anniversaire. Il n'y a pas lieu de passer sous silence cet anniversaire, les regards sur le passé pouvant aider à développer des directives bien fondées pour les activités de l'avenir.

Cette fois, la célébration prévue se veut plus modeste que celle du cinquantenaire. Les personnes intéressées à l'histoire de l'Académie consulteront avec plaisir tant les cours commémoratifs du secrétaire général de l'époque, le regretté professeur René-Jean Dupuy, récemment décédé et dont on peut trouver une nécrologie dans le Bulletin de 1998, et de l'ambassadeur J. H. van Roijen, alors président du Conseil d'administration de l'Académie, que l'ouvrage publié sous le titre Livre jubilaire, 1923-1973 ${ }^{1}$.

Ce livre, préparé par R.-J. Dupuy, contient un certain nombre d'études parmi lesquelles un article très fouillé sur l'histoire, et notamment sur la phase préparatoire, de la main de Stephan Verosta. Il souligne que le premier à proposer une haute école de droit international fut Otfried Nippold, professeur à Berne, dans un article publié en 1907. Il apparaît également que Tobias M. C. Asser a rempli un rôle fondamental ici aussi.

Dans le même ouvrage, on trouvera un article admirable de la main d'Alfred E. von Overbeck, où il donne un aperçu des cours de droit international privé délivrés dans ce premier demi-siècle.

Peut-être pourrait-on s'attendre, à l'occasion de cet anniversaire, à un rapport bien documenté sur les multiples mérites de l'Académie qui, dans leur ensemble, constituent sa «contribution au développement de la science et de la pratique du droit inter-national privé », mais quelques instants de réflexion pourront faire comprendre que tel ne pourra pas être le contenu de cette conférence.

On ne peut que spéculer sur le point de savoir où en serait notre science si l'Académie n’avait jamais été fondée. Dans les arrêts des cours suprêmes, on ne trouve pas de références aux cours publiés dans le Recueil des cours de l'Académie ; il en est de même dans les rapports explicatifs des projets de lois soumis à l'approbation des parlements respectifs. Les perles des connaissances accumulées et des idées avancées suivent des voies plus longues et plus sinueuses pour atteindre les bénéficiaires. Il faut consulter les grands traités de notre matière, les thèses, les mélanges, les notes de jurisprudence dans les grandes revues pour découvrir, surtout dans les notes de bas de page, combien sont nombreuses les références au Recueil des cours. Il n'en reste pas moins que, parfois, on peut se demander pourquoi de telles références font défaut, ou pourquoi elles ne sont pas encore plus fréquentes.

Sur la base de ces références couramment rencontrées, et qui sont le témoin de la large diffusion du Recueil, il paraît légitime de penser que, un peu partout dans le monde, d'une part, l'enseignement du droit international privé s'est enrichi de l'inspiration empruntée au trésor de connaissances qui s'y trouvent stockées et que, d'autre part, les chercheurs se sont vus dispensés de «réinventer la roue» grâce aux résultats si bien présentés du labeur d'une foule de prédécesseurs, avec l'aide, d'ailleurs, d'index très détaillés et pratiques, qui sont mis au point à intervalles réguliers, facilitant nettement les recherches. 
Il n'est pas exagéré de parler de labeur. C’est depuis longtemps que l'Académie jouit d'une grande réputation. Aussi le fait d'être invité à donner un enseignement est-il un honneur pour la personne concernée mais, en même temps, elle se rendra vite compte qu'il s'agit là d'une charge redoutable. A La Haye, il faut se présenter devant un auditoire averti et qui croit être en droit de s'attendre à des développements tout à fait remarquables. Originaires de toutes les parties du monde, les auditeurs forment un jury des plus variés et des plus sévères qu'on puisse s'imaginer.

Ensuite, deuxième épreuve — et c'est bien le cas de le dire —, les conférenciers se souviendront de ce qu'ils se sont enga-gés à présenter leurs propos sous une forme appropriée, et dans les meilleurs délais, à l'éditeur de la maison pour que, élégamment imprimées, leurs brillantes idées soient bientôt accessibles à tout le monde — vieux routiers dans le domaine, plutôt critiques, ou jeunes personnes intéressées, et parfois un peu naïvement admiratrices - et ce pour l'éternité, parce que scripta manent. Heureux ceux des enseignants qui ont achevé leur texte avant d'arriver à La Haye! Quelle que soit la renommée des enseignants, les cours imprimés seront toujours le résultat d’un effort extrême.

Depuis soixante-quinze ans, avec une interruption de sept ans (1940-1946) à cause de la seconde guerre mondiale, une légion de grands officiers, une foule de géants de notre discipline s'est rendue à La Haye, mais, en consultant la liste des cours publiés depuis 1923, on ne manquera pas de se poser la question de savoir pourquoi tel ou tel savant éminent n'y a pas laissé trace, cela d'autant plus que d'autres ont enseigné à plusieurs reprises, comme J.-P. Niboyet, quatre fois, Hans Lewald, quatre fois, et B. A. Wortley, de même. Par exemple, on n’y trouvera ni le nom illustre d'Ernst Rabel, ni de Paul Lerebours-Pigeonnière, ni de Joseph Beale, ni de Pierre Wigny, ni de René Savatier, ni de J. H. C. Morris, ni de J. Kosters, et ainsi de suite: George Melchior, Hans Dölle, Erwin Riezler, Martin Wolff, Pierre Lepaulle, Léon Julliot de la Morandière, Hessel Yntema, Paul-Heinrich Neuhaus, Johannes Offerhaus, Kurt Nadelmann, Max Pagenstecher, pour ne mentionner que quelques-unes des personnes décédées, et ne pas attirer l'attention sur des personnes encore vivantes. Dans chaque cas, on se pose la question de savoir pourquoi. Des problèmes linguistiques, étant donné que, avant 1940, tous les enseignants devaient s'exprimer en français et, depuis 1947, soit en français soit en anglais ? Des problèmes financiers ? Des problèmes de santé ? Des rivalités académiques ? A-t-on négligé de les inviter ? ou ont-ils boudé l'Académie ? ou encore ont-ils disparu dans la nuit de l'oubli, en n'ayant pas réussi à présenter leur texteà l'éditeur, ce qui d'ailleurs n'exclut pas que viva voce ils auraient fait une grande impression sur l'auditoire ? Nous ne le saurons jamais.

Dommage, mais ce regret ne peut que renforcer la raison d'être fiers de ce corps d'enseignants illustres qui, au prix de grands efforts, ont apporté leur contribution à la grande réputation de l’Académie. 
Fierté, certes, mais on ne peut s'empêcher de relever que, parmi ces enseignants, les femmes ont dû mettre beaucoup de temps pour pouvoir se présenter. Pour le droit international public, nous rencontrons $\mathrm{M}^{\mathrm{me}}$ Susanne Bastid, à plusieurs reprises même, la première fois en 1951, et d'autres encore, parmi lesquelles $\mathbf{M}^{\text {me }}$ Denise Bindschedler (1968), $M^{\text {me }}$ Rosalyn Higgins (1982 et 1991), actuellement juge à la Cour internationale de Justice, première femme à assu-mer cet office, et $\mathbf{M}^{\text {me }}$ Geneviève Burdeau (1988), le nouveau secrétaire général de l'Académie dès le début de l'année prochaine; mais, pour ce qui concerne le droit international privé, on a dû attendre $\mathrm{M}^{\mathrm{me}} \mathrm{I}$. H. P. Diederiks-Verschoor, en 1981. Heureuse-ment, elle n'est pas restée la seule ! Depuis, $\mathbf{M}^{\text {mes }}$ de Maekelt, Prott, Kay, Gaudemet-Tallon, Borrás, Brilmayer, Pérez Vera et Ginsburg ont pu monter sur l'estrade.

Il paraît légitime de penser que nombreux sont les enseignants qui ont fait une forte impression sur les auditeurs. Aussi ceux-ci, une fois rentrés dans leurs pays, et devenus avocats, juges, notaires, fonctionnaires dans un ministère, ou conseils dans une entreprise, se souviendront-ils des leçons de La Haye et du Recueil, dans lequel ils trouveront le texte imprimé des cours de leur session, venant côtoyer celui de tant d'autres cours comparables. Les auditeurs auront tout intérêt à être tenus au courant de la parution des nouveaux tomes du Recueil et des nouveaux catalogues, ne serait-ce que désormais par Internet.

Je me rends compte que ma tâche est délicate. Je ne vous le cache pas. Faut-il vraiment que, dans un langage diplomatique — cela s'entend —, j'émette des jugements de valeur pour établir une hiérarchie entre les porteurs d'une médaille d'or des Olym-piades du droit international privé ? Je sais parfaitement que je ne suis pas le juge très chevronné qu'il faudrait. Et même l'opinion d'un tel juge n'aurait qu'une valeur relative. Ses pairs pourraient très bien être d'un autre avis. S'il n'y a qu'une seule vérité, elle semble être audelà de la portée des êtres humains, même les plus doués.

Il n'en reste pas moins que celles et ceux qui sont appelés à donner des cours profitent largement des travaux de celles et de ceux qui les ont précédés, vu les nombreuses références aux cours publiés antérieurement. On cherche des appuis dans le travail des autres. Ainsi le progrès escompté est-il le fruit des efforts d'une équipe qui s'étend dans le temps. On se hisse, pour utiliser une image bien connue, sur les épaules des géants du passé.

Un autre facteur commun aux enseignements dispensés à l'Académie pourrait être plus important encore, bien que plus difficile à prouver. Si, normalement, l'esprit de clocher d'une personne n'est pas susceptible de susciter des compliments de la part de ses interlocuteurs, il n'en est pas de même de l'esprit du clocher du Palais de la Paix. Ce clocher est bien connu un peu partout dans le monde ; il symbolise la mission confiée à la Cour permanente de Justice internationale d'abord et à la Cour internationale de Justice ensuite. Ce clocher invite les enseignants qui sont appelés à La Haye à accomplir leur tâche dans un esprit qui soit en harmonie parfaite avec cette mission à laquelle l'Académie participe. C'est ce à quoi peuvent s'attendre les auditeurs. Ils se rendront compte du fait que ce n'est pas par hasard ou pour une raison banale que l'Académie a son siège à l'intérieur des grilles qui entourent le Palais de la Paix. Pour ce qui concerne le droit international privé, et pas moins que pour le droit international public, la proximité de la Cour pousse à le développer dans un sens qui est favorable aux bons rapports internationaux. Aussi semble-t-il légitime de soupçonner un tel dénominateur commun.

Dès 1930, Etienne Bartin, qui, dans l'histoire du droit international privé s'est acquis la réputation d'un " particulariste ", en est conscient, lorsqu'il dit, à la fin de son cours sur «La doctrine des qualifications et ses rapports avec le caractère national du conflit de lois ${ }^{2}{ }^{2}$ : 
«Que devient, dans ces conditions, l'esprit international sans lequel il semble difficile de participer à l'enseignement donné à La Haye. Si contraires que soient les apparences, on n’hésite pas à répondre que l'esprit international n’a pas disparu de ces leçons. »

Il ne renie aucunement les obligations internationales, mais souligne que:

«Ces obligations touchent ici, par leur objet, à ce qu'il y a de plus national et de plus individuel dans un Etat, sa législation, sa classification des institutions, les rapports que ces institutions soutiennent entre elles. L'étendue de ces obligations ne saurait être déterminée de même pour les différents Etats. Il y a là une différence irréductible entre eux, qu'il ne semble pas que l'esprit international puisse jamais faire disparaître. »

Il poursuit : «L'avenir de l'esprit international, selon moi, est ailleurs.» Et de parler de conventions appropriées, dont il dit : « ce n’est pas impossible mais à ... une condition sine qua non : c'est que ces conventions soient minutieusement élaborées... »

Ces observations pourraient nous laisser perplexes, raison pour laquelle je me permets de citer également Henri Batiffol, qui, dans son cours de 1959, semble réagir au passage cité de Bartin :

«On ne peut méconnaître que les différenciations de structures entre les Etats engendrent des différenciations inévitables entre leurs systèmes de droit international privé. Le problème est de travailler à réduire les différences des résultats et on peut aller loin dans cette voie, beaucoup plus loin que les particularistes ne le laissent croire. ... L'avenir du droit international privé est donc dans l'étude méthodique et persévérante du droit comparé. Cette voie est celle qui permettra d'arriver à un droit plus juste et plus efficace : les incohérences et les contradictions mènent finalement à l’inefficacité et ne sont pas signe de justice. Il y a une cohérence du bien.» ${ }^{3}$

Comment pourrait-on mieux expliquer ce qu'est cet esprit interna-tional ?

Probablement, les enseignants ne manqueront pas de faire état du développement du droit international privé dans leurs pays respectifs, d'en souligner les éléments qui mériteraient une attention particulière des experts étrangers et qui pourraient inspirer les responsables d'autres pays. Ils sont censés apporter leur contribution dans un esprit de coopération. Cela ne les empêchera pas d'exposer les difficultés qu'ils ont rencontrées, au contraire. Une fois de retour de la tâche accomplie à La Haye, ces enseignants exerceront dans un esprit international une influence certaine sur le développement ultérieur du droit international privé dans leur pays. Ils se souviendront des échanges de vues qu'ils ont eus avec des collègues originaires d'autres pays et qui se trouvaient confrontés à une tâche similaire, ainsi que des rencontres avec les étudiants qui ont suivi leurs cours sur la base des conceptions juridiques acquises dans leurs pays respectifs.

Essayons maintenant d'explorer les résultats des activités que l’Académie a développées depuis 1923. Il nous faudra bien faire une sélection.

Laissons de côté le Centre d'étude et de recherche de droit international et de relations internationales, lequel a été très important, et l'est toujours, tout d'abord pour le droit international public. Sa première session s’est déroulée en 1957. 
Il en est de même du Programme extérieur qui, depuis 1969, est organisé chaque année dans un pays économiquement défavorisé, c’est-à-dire soit en Afrique, soit en ExtrêmeOrient, soit en Amérique latine. Cependant, ce programme contient parfois des éléments de droit international privé. L’idée du programme extérieur reflète le souci constant du Curatorium, depuis fort longtemps, de faire rayonner l'Académie réellement à une échelle mondiale et de diffuser la connaissance du droit international dans toute la mesure possible en faveur des juristes originaires des pays pauvres pour qui il serait trop onéreux de se déplacer à La Haye.

Aujourd'hui, notre concentration va porter sur ce qui s'est passé pendant tous ces étés consécutifs à La Haye. Il est clair que plusieurs choix seraient possibles. Dans une première partie, il s'agira de donner un tableau d'ensemble, avec tous les accents subjectifs qui sont inévitables. Dans une deuxième partie, nous voudrions essayer de retracer l'historique de deux thèmes que la majorité des orateurs n’aura pas négligés, à sa-voir l'autonomie des parties à un contrat, et les lois dites «lois de police».

\section{Tableau des cours}

En droit international privé, étant donné le sujet de la présente communication, la distinction s'impose entre, d'une part, la partie générale et, d'autre part, les thèmes plutôt spécifiques. Un grand nombre de cours importants ont pour objet cette partie générale, dans une acception plus ou moins large. Ce sont les cours généraux. Depuis longtemps, un cours général est prévu au programme de chaque année. Normalement, ces cours généraux sont confiés à des vétérans renommés et qui ont déjà fait preuve de leurs qualités à l'occasion d'un autre cours.

D'autres cours ont pour objet un thème spécifique, mais qui, indiscutablement, trouve sa place dans l'ensemble de la partie générale, tels la réciprocité, le phénomène du droit étranger avec toutes les ques-tions qu'il soulève, le conflit mobile, la question préalable et le renvoi.

D'autres cours encore ont pour objet un thème qui correspond avec une section du droit privé, tels le mariage, la filiation, les biens culturels, la responsabilité des produits et le transport aérien.

Inévitablement, nous ne pouvons que nous borner à quelques observations générales en guise de points de repère dans ce paysage varié.

\section{Les grands continents du globe du droit international privé}

Dès le début, le Curatorium s'est rendu compte que l'Europe continentale, aussi intéressante que soit son histoire, ne représente que l’un des grands courants de pensée dans le domaine du droit international privé, avec toutes ses variations, bien entendu. Le monde anglo-saxon peut prétendre à une place à lui, et même une double place, parce qu'il n'est pas indiqué de confondre les Etats-Unis etle Royaume-Uni. On a bien pris soin de s'assurer que les Anglo-Saxons aient leurs porte-parole les plus distingués à l'Académie.

$\mathrm{Si}$, en 1924, Antoine Pillet est venu donner un cours sur «La théorie continentale des conflits de lois ", H. H. L. Bellot a enseigné la même année, en français, sur « La théorie anglo-saxonne des conflits de lois ». Une ligne de conduite s'est fixée depuis. Très régulièrement, des Britanniques et des Américains ont été invités à apporter leurs points de vue à côté des Européens continentaux. On trouve même un cours comparatif du droit international privé en Angleterre et aux Etats-Unis, par R. H. Graveson (1960). 
Mais même une telle politique aurait été en deçà de la vocation mondiale de l'Académie et on n'a pas négligé de faire venir des représentants du monde communiste à l'époque où le pouvoir politique et militaire des Etats communistes, avec l'Union soviétique en tête, semblait pouvoir assurer qu'une culture juridique nouvelle et originale fût en train de prendre naissance sur la base de l'idéologie marxiste-léniniste. Apparemment, on a voulu éviter à tout prix qu'une partie importante du monde eût le sentiment bien fondé d'être tenue à l'écart. Peut-être l'Académie a-t-elle, en faisant de la sorte, contribué à sauvegarder à l'échelle mondiale la crédibilité de la Cour internationale de Justice où l'un des sièges était réservé à un juge originaire de l'Union soviétique. Il n'en reste pas moins que les experts invités n’ont pu venir sans y avoir été autorisés par le régime suspicieux de l'Etat concerné. G. I. Tunkin a pu faire partie du Curatorium et donner, par quatre fois même, un enseignement en droit international public. L. A. Lunz, de Moscou, a pu faire son apparition en 1965, S. N. Lebedev en $1977^{4}$, M. M. Bogouslavski en $1981^{5}$, ainsi que quelques Polonais, Tchèques et Yougoslaves, citoyens d'Etats qui, à l'époque, étaient un peu plus ouverts à l'Ouest.

On n'a pas négligé non plus d'inviter des experts provenant du monde islamique, porteur d'une grande et ancienne culture et qui, depuis longtemps et de plus en plus, réclame sa part dans la gestion politique de la planète. En 1937, C. Cardahi a donné une série de leçons sur « La conception et la pratique de droit international privé dans l'Islam» ${ }^{6}$, et après lui, outre S. Mahmassani qui s'est concentré plutôt sur le droit international public (1966), M. Charfi, en 1987, J. Déprez, en 1988, et A. Moulay Rachid, en $1997^{7}$.

On ne peut s'empêcher de constater que, jusqu'à présent, on n'a guère réussi à trouver de représentants des grandes cultures de l'Extrême-Orient ou de l'Inde pour informer les auditeurs de l'Académie, ainsi que les lecteurs du Recueil, sur les questions de ce que nous avons pris l'habitude de dénommer le droit international privé, et les réponses possibles, et qui trouvent leurs racines dans ces -cultures. Si le rôle du droit dans la société se différencie de celui qui est courant dans les pays occidentaux, on aimerait savoir comment les problèmes qui, du point de vue des Occidentaux, se présentent sont perçus et quelles sont les solutions à y apporter et qui puissent satisfaire les Orientaux. K. R. R. Sastry ${ }^{8}$ et K. N. Jayatilleke ${ }^{9}$, de Ceylon, ont été de rares experts de cette partie du monde que l'Académie a pu accueillir. En 1966, le premier a enseigné sur l'hindouisme et le droit international, tandis que l'année suivante le deuxième a donné un cours sur les principes du droit international selon la doctrine bouddhiste. Haopei Li, de Beijing, est venu en $1990^{\text {10, }}$ S. Murase, de Tokyo, en 1996, et on aura écouté et on lira avec le plus vif intérêt les enseignements donnés cette année par les profes-seurs Xu, de Shanghai, et Sakurada, de Kyoto.

Dans une zone contiguë, en ce sens que la différence de la culture sous-jacente fait également soupçonner que certaines questions se posent nécessairement d’une manière originale et nouvelle du point de vue des Occidentaux, on peut signaler les leçons de $\mathrm{Ph}$. Francescakis sur les problèmes de l'Afrique noire indépendante et de I. Szászy sur le conflit de lois interpersonnel dans les pays en voie de développement, et, plus récemment, les cours de U. U. Uche etA. K. Boye sur les conflits de lois dans les pays africains au sud du Sahara, et celui de $\mathrm{M}^{\mathrm{me}}$ Alegría Borrás sur les ordres plurilégislatifs ${ }^{11}$. 
Il y a la diversité de culture et de pensée juridique que le Curatorium a pris soin de faire refléter dans les programmes, mais il y a des diversités d'un autre ordre encore qui trouvent leur écho. On s'est rendu compte que, en matière de droit international privé, les problèmes se posent surtout dans le cadre des ordres juridiques étatiques et que c'est dans ce cadre que les organes compétents étatiques, qu'ils soient législatifs ou judiciaires, sont appelés à y trouver des solutions adéquates. Voilà une raison pertinente pour inviter des experts à venir faire état du droit international privé de leur pays, et notamment de son développement à la suite, par exemple, d'une opération récente de codification. Ces cours peuvent constituer une source précieuse d'informations sur le droit de tel ou tel pays, mais, au-delà de cela, présenter des matières premières pour découvrir combien sont variés, et combien sont similaires, les problèmes qui peuvent se poser. Ils permettent de vérifier la validité de tel ou tel élément de la théorie générale. Soulignons que c’est surtout dans les tomes du Recueil d'avant 1940 qu'on trouvera un certain nombre de tels enseignements.

Cela dit, il faut bien signaler que certains autres cours, en fait, ne présentent qu'un aperçu du droit international privé du pays de l'enseignant, si on se réfère aux références à des dispositions législatives, àla jurisprudence ainsi qu’à la littérature. Cela s'explique en partie par des problèmes linguistiques, problèmes non négligeables il est vrai dans le domaine du droit international privé. Mais faire du droit comparé n'est-il pas indispensable pour réellement avancer en droit international privé, comme l'a souligné si souvent Henri Batiffol ? Si cela est vrai, il faut bien avouer que la tâche de vouloir rester au courant du droit international privé national peut déjà constituer une charge susceptible d'absorber toute l'énergie disponible. Mais, parfois, il est difficile de ne pas penser que l'orateur, animé de l'esprit de cet autre clocher, a estimé que son droit international privé national était le seul digne à entrer en ligne de compte pour être exposé à l'Académie.

Ce qui est vrai de l'utilité d'être renseigné sur le droit international privé des Etats isolés peut valoir également pour un groupe d'Etats animés par le sentiment d'appartenir à une communauté à cause de leur situation géographique et de leur histoire. Tel est le cas pour la Scandinavie - voir le cours de A. Philip ${ }^{12}$ — comme pour l'Amérique latine. Malgré les dimensions de ce continent, très tôt dans le XIX ${ }^{\mathrm{e}}$ siècle le droit international privé a fait l'objet de négociations en vue de la mise au point de conventions et cette coopération s'est poursuivie depuis. Récemment encore, nous avons pu enregistrer des progrès considérables. Régulièrement, des experts de grande renommée sont venus à La Haye pour présenter de la coopération un rapport circonstancié et enrichi de leurs réflexions personnelles, tels Haroldo Valladão, $\mathbf{M}^{\text {me }}$ Tatiana de Maekelt, Manuel Adolfo Vieira, Gonzalo Parra-Aranguren, actuellement juge à la Cour internationale de Justice, et Antonio Boggiano ${ }^{13}$.

Cette famille latino-américaine évoque les problèmes qui se présentent à l'intérieur d'un Etat qui consiste en plusieurs unités qui, chacune, doit son identité à un ordre juridique à elle. On a parlé de systèmes plurilégislatifs pour cerner des questions de droit quasi international privé, de droit interrégional privé plutôt. Ces questions ont fait l'objet de plusieurs cours, à savoir celui de P. Arminjon (1949), de B. W. Cowles (1949), de R. H. Graveson (1974) et de $\mathrm{M}^{\mathrm{me}}$ Alegría Borrás (1994). Il y a une certaine ressemblance avec le problème de la coexistence des droits confessionnels et des droits laïcisés dans les relations privées internationales, objet du cours de P. Gannagé (1979). 
L'intégration européenne a eu, et a progressivement, pour effet que les Etats membres développent des rapports quasi familiaux. L’interdépendance à laquelle ils ont consenti, bon gré mal gré selon les circonstances, entraîne des effets dans le domaine du droit international privé. Les rapports internationaux à l'intérieur de l'Union européenne sont devenus un peu moins internationaux que d'autres. Aussi y a-t-il lieu d'examiner cette évolution, ce qui explique que des cours spéciaux ont eu lieu, à savoir ceux donnés par Giorgio Badiali (1985), votre serviteur (1992), Marc Fallon (1995) et $\mathrm{M}^{\mathrm{me}}$ Elisa Pérez Vera (1996).

\section{L'évolution dans le temps et les rapports avec le droit international public}

Le droit comparé dans l'espace trouve un parallèle dans le droit comparé dans le temps. Et, effectivement, l'histoire du droit international privé mérite une attention particulière. Nombreux sont les cours où on trouve des paragraphes consacrés à l'histoire de la science du droit international privé ou des exposés d'affaires célèbres. Moins nombreux, ou plutôt rares, trop rares même, sont les cours délivrés par des professeurs de droit qui y font preuve d'être, en outre, de vrais historiens du droit. C'est à juste titre qu'on s'appuie toujours sur les cours de Max Gutzwiller (1929) et d'Eduard Maurits Meijers (1934). Il faut signaler encore le cours de Giuseppe Barile (1965) et celui d'Erik Jayme (1982), et relever des chapitres dans des cours généraux comme ceux de Rodolfo de Nova (1966), de Kurt Lipstein (1972) et de Friedrich K. Juenger (1983) ${ }^{14}$. On dirait que certaines lacunes de l'ensemble des cours pourraient être comblées si on invitait de vrais historiens du droit à présenter les résultats de leurs recherches dans le domaine du droit international privé. Dans le traité autorisé de Gerhard Kegel, on trouve deux portraits, deux seulement, et choisis par l'auteur à bon escient, à savoir celui de Friedrich Carl von Savigny et celui d'Henri Batiffol. Ces autorités ont marqué leur époque, ce qui justifie les recherches historiques ainsi que juridiques approfondies et qui les placent dans le cadre des grands courants de pensée de leur temps. L'Académie peut prétendre être le cadre approprié pour en présenter les résultats. Il en est de même d'autres personnalités comme Joseph Story, Pasquale Stanislao Mancini et Tobias Michael Carel Asser. Il s’agit de repérer des spécialistes qui sont juristes et historiens à la fois : ils sont plutôt rares.

Depuis la naissance du droit international public au XVII ${ }^{\mathrm{e}}$ siècle, les rapports entre cette nouvelle branche du droit, d'une part, et le droit international privé, d'autre part, ont fasciné, et divisé, les esprits. Aussi ne sera-t-on pas étonné d'apprendre que des cours ont été consacrés spécifiquement à ce thème, et notamment par B. A. Wortley (1954), W. Riphagen (1961) et E. Hambro (1962). Ce thème présente un lien indiscutable avec celui de la réciprocité, qui a fait l'objet de deux cours, à savoir ceux de J.-P. Niboyet (1935), sur la réciprocité dans les traités diplomatiques de droit international privé, et de Paul Lagarde (1977), sur la réciprocité en général. 


\section{Le débat atlantique}

Dans ce qui précède, on a déjà souligné que les Britanniques et les Américains ont toujours été invités à présenter leurs conceptions à l'Académie, et ce pour de bonnes raisons. Depuis quelques décennies, ces raisons sont d'autant plus fortes que, du côté américain, plusieurs auteurs ont fait des efforts très sérieux pour renouveler totalement la théorie du droit international privé. Ce faisant, ils ont surtout pensé à la situation prévalant aux Etats-Unis où l'élément d'extranéité qui caractérise les problèmes de droit international privé consiste en un lien avec un autre Etat de la Fédération, mais leurs idées s’appliquent sans grande difficulté aux cas vraiment internationaux. Ces théories américaines modernes, qui présentent une grande variété entre elles, sont le résultat d'efforts intellectuels tout à fait remarquables. Elles ont constitué, et constituent toujours, un défi, un autre "défi amé-ricain », pour les Européens.

Les Américains qui représentent ce nouveau courant ont eu maintes occasions pour envoyer leurs porte-parole à La Haye :E. E. Cheatham en 1960 ; Willis Reese, de Columbia, New York, premier responsable du Restatement Second du 23 mai 1969, qui a été ici même en 1964 et, de nouveau, pour le cours général, en 1976 ; Albert A. Ehrenzweig, qui avait eu sa formation de juriste dans son pays d'origine, l'Autriche, avant d'émigrer aux Etats-Unis, et qui donna le cours général en 1968, tout comme le fit D. F. Cavers en 1970 ; F. K. Juenger, qui a eu sa formation de juriste en Allemagne, donc lui aussi dans la tradition de l'Europe continentale, avant d'émigrer aux Etats-Unis, et qui a donné le cours général en 1983 ; R. J. Weintraub, qui, en 1984, a traité des contrats, et $\mathbf{M}^{\mathrm{me}}$ Herma H. Kay, qui, en 1989, a présenté son cours sous le titre «A Defense of Currie’s Governmental Interest Analysis »; Peter Hay, en 1991, et, en 1995, $\mathbf{M}^{\text {me }}$ Lea Brilmayer, de New York ; et encore deux auteurs de cours généraux, à savoir, en 1994, A. F. Lowenfeld et, en 1996, Arthur von Mehren, de Harvard. En présence d'une délégation américaine si nombreuse, on peut deviner combien ces cours forment une source des plus précieuses d'informations sur ces nouvelles théories.

En Europe, on s'est rendu compte de la nécessité de relever ce défi américain. Un certain nombre d'auteurs européens ont été fascinés par les nouveautés d'outre-Atlantique. A l'Académie, les auditeurs ont eu l'occasion d'écouter leurs commentaires. Il y a eu des cours spécifiques, comme ceux de Gerhard Kegel, de Cologne, dès 1961; de Dimitrios Evrigenis, de Thessalonique (1966); de Bernard Audit, de Paris (1984); d'Andreas Bucher, de Genève (1993), et de Th. M. de Boer, d'Amsterdam (1996). On peut ajouter qu'il n'est guère possible de trouver un cours général qui n’y consacre pas de développements substantiels. De bons exemples sont ceux de Kurt Lipstein, de Cambridge (1972), de A. Ferrer-Correia, de Coimbra (1975), et de F. Vischer, de Bâle (1992).

Le moins qu'on puisse faire remarquer, c'est que les continentaux, en se rendant compte de leur responsabilité scientifique, ont fait des efforts sérieux pour repenser leurs idées traditionnelles sur la base des critiques qui constituent ce défi américain. Ils ont découvert des parallèles et dégagé des éléments qui sont susceptibles d’être repris, tels que l’idée de la " governmental policy», politique gouvernementale. Cette idée peut avoir contribué à éclaircir la notion de « loi d'application immédiate », et notamment celle de « loi de police » qu'on retrouve désormais, en Europe continentale, comme titre de l'article 7 de la Convention CE de Rome du 19 juin 1980 sur la loi applicable aux obligations contractuelles. Nous en traiterons ci-après. 
A l'inverse, les Américains semblent être peu enclins à s'inspirer des développements de la doctrine continentale, à quelques exceptions près, comme Peter Hay et F. K. Juenger ${ }^{15}$. Peut-être, citoyens d'une superpuissance et en outre d'une ancienne colonie, ne leur convient-il pas de se renier, un peu comme des protestants qui reconnaîtraient un mérite quelconque au pape. Quoi qu'il en soit, le débat bat toujours son plein.

\section{Les cours généraux}

Le moment est venu d'évoquer les cours généraux. Tâche délicate! Il ne s'agit pas d'énumérer tous les protagonistes qui en sont les auteurs, tandis que le fait de faire une sélection reviendrait à porter un jugement de valeur en distinguant entre ceux qui sont très bons

et ceux qui sont meilleurs encore, et je ne serais pas le mieux placé, ni pour mettre au point un classement, ni pour formuler une obser-vation critique. Passons donc sous silence en tout cas tous ceux qui, heureusement, sont toujours parmi nous. Nombreuses sont les personnes ici présentes qui connaissent les cours admirables d'un passé récent.

D'avant-guerre, on cite toujours les cours d'Antoine Pillet, de Roberto Ago, de Jacques Maury et de Hans Lewald, ainsi que ceux de L. Raape, de B. Nolde et de E. Frankenstein, qui constituent des jalons dans le développement de la théorie du droit international privé.

Après la réouverture de l'Académie, en 1947, pendant les premières décennies, ce sont B. A. Wortley (1947 et 1958), Henri Batiffol (1948, 1959, 1967, 1973), R. H. Graveson (1960, 1963, 1974), Willis Reese (1964, 1976), Wilhelm Wengler (1961), M. K. Yasseen (1962, 1965, 1976), Rodolfo de Nova (1966), G. Sperduti (1967), Albert A. Ehrenzweig (1968), G. van Hecke (1969), D. F. Cavers (1970), K. Lipstein (1972), O. Kahn-Freund (1974), et Edoardo Vitta $(1969,1979)$ qui ont donné le cours général, lequel, pour la majorité d’entre eux, n'était pas leur seul exploit à l'Académie. On ne peut trop louer l'étendue et la profondeur de leurs recherches, la richesse de leurs idées ainsi que la clarté de leurs exposés; bien entendu, cela sans méconnaître les mérites de tous les autres, ceux qui les ont précédés comme ceux qui sont venus après pour donner leur cours général.

Beaucoup d'entre eux ont fait preuve de leur qualité de polyglotte, ce qui leur a facilité des recherches comparatives. Ils ont pu appuyer leurs idées sur des connaissances du droit international privé de plusieurs Etats. On peut pressentir leurs efforts tenaces et leur détermination pour essayer de progresser et de ne pas se contenter d'un tableau d'ensemble du droit international privé dans l'état où il se trouvait. Cela vaut pour les cours généraux d'un passé récent comme pour ceux de la génération qui n’est plus.

Le cours général constitue le noyau d'une session et, de ce fait, détermine pour une grande partie son succès. Si dix leçons orales ne suffisent pas pour couvrir l'ensemble de la discipline, l'éditeur est plus indulgent en ce qu'il accepte des textes qui se présentent comme des traités plus ou moins complets. En effet, plusieurs enseignants peuvent s'enorgueillir d'avoir rempli tout un tome du Recueil ${ }^{16}$. Les lecteurs leur en sauront gré. 
Selon une acception du droit international privé qui est courante dans certains pays, les problèmes de compétence judiciaire et ceux de la reconnaissance et de l'exécution de jugements étrangers constituent une branche du droit distincte ${ }^{17}$. Quoi qu'il en soit, de plus en plus souvent, les cours généraux ne laissent pas ce domaine à l'écart, heureusement parce qu'il y a des liens multiples avec le droit international privé au sens le plus étroit (voir, par exemple, le cours général de Georges Droz (1991) et celui de F. Vischer (1992)). A côté de cela, on trouve, depuis le début des activités de l'Académie, des cours qui ont pour objet soit la com-pétence, soit la reconnaissance et l'exécution, soit les deux.

Un thème plus restreint et relevant de ce domaine est celui des immunités. Le tout premier tome du Recueil contient un cours d'André Weiss (1923) sur la compétence ou l'incompétence destribunaux à l'égard des Etats étrangers. Le sujet n'a rien perdu de son actualité (voir les cours de J.-F. Lalive, en 1953, et de Peter Trooboff, en 1986 — et d'autres cours encore).

Mis à part ce sujet, largement dominé par le droit international public, la compétence judiciaire est un thème de toute première importance. La vérité juridique, en fait, est loin d'être universelle; elle est, pour une grande partie du moins, celle formulée par les tribunaux, et, normalement, ceux-ci relèvent de l'ordre judiciaire d'un Etat. Aussi n'est-il aucunement indifférent de savoir quel tribunal sai-sir, et est-il, par conséquent, de première importance de savoir à quelles conditions les tribunaux d'un Etat donné peuvent être saisis.

Dans la tradition du droit international privé anglais, par exemple, la compétence judiciaire est un thème qui domine la matière. Il en est de même aux Etats-Unis où la norme constitutionnelle de due process et celle de full faith and credit ont assuré à la Cour suprême un rôle prédominant dans le développement des critères de compétence dans les rapports entre les Etats de la Fédération, et, indirectement, dans les rapports internationaux. Les orateurs anglais et américains n’ont pas manqué de s'étendre sur les questions de "jurisdiction », au sens de compétence judiciaire. Ainsi, H. C. Gutteridge (1933). Des quatre cours enseignés par F. A. Mann, l'un des nombreux grands juristes allemands qui ont dû quitter le continent européen pour s'insérer dans les rangs des juristes anglo-saxons et américains, deux cours $(1964,1984)$ ont eu pour objet la compétence. Il en est de même du cours général de M. Lowenfeld (1994).

Cependant, les continentaux ne se sont pas tenus à l'écart, comme le prouvent les cours de N. Fragistas (1961), de A. Miaja de la Muela (1972) et de Paul Lagarde (1986). Des problèmes spécifi-ques ont retenu l'attention, comme celui des clauses d'élection de for, par J. Jodlowski (1974), et celui des liens entre la compétence judiciaire et la compétence législative, par J. D. Gónzalez Campos (1977).

En 1980, Arthur von Mehren a consacré son cours à la reconnaissance et à l'exécution de jugements étrangers, et notamment aux conditions, tout comme, en 1997, Konstantinos D. Kerameus, qui, notamment, s'est concentré sur l'exécution.

Cependant, il n'y a pas que les problèmes de compétence juridictionnelle et ceux de reconnaissance et d'exécution. L'appli-cation, d'office ou non, des règles de droit international privé et de la loi étran-gère applicable, la preuve de la loi étrangère, la preuve en général,la légalisation, la signification et la notification des actes judiciaires et extrajudiciaires sont autant de thèmes qui méritent l'attention. Qu'on pense aux conventions de La Haye qui relèvent de ce domaine. Cette attention, ils l'ont tous eue, bien que de manière inégale. 
Si important que soit, pour la doctrine du droit international privé, le chapitre de la procédure par-devant un tribunal étatique et des effets que produisent les jugements en dehors du pays où ils ont été rendus, le contentieux du commerce international se trouve être soumis pour une grande partie aux tribunaux arbitraux. Pas moins de douze cours ont eu l'arbitrage pour objet, et encore plus si on y ajoute les nombreux cours relatifs aux contrats d'Etat et les investissements internationaux, et au contentieux concernant de tels contrats. Parmi les grands noms des enseignants spécialistes de cette matière, il y a lieu de mentionner celui de Berthold Goldman, de Paris, protagoniste du débat sur la lex mercatoria $^{18}$.

Les notaires, les huissiers, les officiers de l'état civil et les autres officiers ministériels qui remplissent un rôle si important dans le commerce juridique national aussi bien qu'international n'ont pas eu l'attention qu'ils méritent, semble-t-il, ce à la différence des officiers consulaires.

La faillite, qui fait l'objet de plusieurs conventions internationales plus ou moins réussies, ne devra pas être perdue de vue, ne serait-ce qu'à cause de ses intérêts théoriques et pratiques.

\section{D'autres thèmes}

Nombreux sont les autres thèmes qui ont retenu l'attention. Il y en a qui ont pour objet un élément de la partie générale, tels la loi étrangère, le conflit mobile, la question préalable, l'adaptation.

D’autres, également d'un intérêt indiscutable, se rapportent à une partie du droit privé, tant dans le domaine du droit de la famille que dans celui du droit patrimonial. La liste serait trop longue pour qu'il soit possible de les énumérer. Ce qui est regrettable, à cause de la valeur de ces cours en tant que cours spécialisés aussi bien qu’à cause des mérites de leurs auteurs qui ne doivent pas être escamotés, ce d'autant plus que ces cours spécialisés contiennent des idées touchant aux problèmes de la partie générale.

Pour ce qui concerne le droit de la famille, les mérites respectifs des points de rattachement les plus importants, la nationalité et le domicile - pendant de longues années un sujet de débat inépui-sable —, ont fait l'objet de toute une série de cours. Il en est de même des grands sujets comme le mariage et le divorce, la filiation et l'adoption, la protection des faibles, les régimes matrimoniaux et les successions.

Tout un groupe de cours nous informe sur tous les aspects possibles de la personnalité morale et des sociétés.

Nombreux sont les cours sur les contrats, à savoir les contrats en général aussi bien que des contrats spécifiques comme la vente, l'assurance et le transport. On constatera un certain dés-équilibre dans la mesure où le transport aérien a été favorisé par rapport aux transports maritime, ferroviaire et routier. Les aspects de droit privé des services postaux internationaux et des télécommunications sont restés quelque peu dans l'ombre, jusqu'à présent, mais, cette année, le Centre d'étude et de recherche ne manquera pas de les englober dans son programme. En général, le droit matériel uniforme et les conditions générales comme les pratiques du commerce international devraient ne pas passer inaperçus.

La responsabilité civile n'a pas été négligée, aucunement, surtout si on se rend compte que cette responsabilité constitue le champ de prédilection des théories américaines.

Les paiements internationaux ont fasciné nombre d'experts. Ils ont donné des cours sur la clause-or, il y a quelque temps, et plus récemment, sur le droit monétaire, le droit bancaire et d'autres encore si on ne perd pas de vue les cours relevant plutôt du droit international public. 
Pendant longtemps, les biens n’ont pas bénéficié d'un traitement de faveur, malgré, par exemple, l'intérêt suscité par les clauses de réserve de propriété dans le commerce international, ou bien par le crédit-bail (leasing), ou par le trust, mais, récemment, des lacunes ont été comblées par K. Kreuzer, J. Voulgaris et D. W. M. Waters ${ }^{19}$, de manière remarquable.

Le régime des moyens de transport international, y inclus celui des conteneurs, soulève toujours des questions. Il en est de même de la cession de créances qui est de plus en plus importante pour le financement des investissements. La question se pose de savoir dans quelle mesure l'autonomie de la volonté pourrait s'étendre à cette matière, ou, davantage encore, au domaine des droits réels et des droits patrimoniaux en général.

Et on se félicitera de voir que le régime des biens culturels, toujours un sujet de grande actualité, a fait l'objet d'exposés très fouillés (voir le travail de $\mathrm{M}^{\text {mes }}$ Prott et Galenskaya et de Kurt Siehr ${ }^{20}$ ).

La concurrence ne cesse de soulever nombre de questions dans le domaine du droit international privé aussi bien que dans d'autres domaines du droit. La propriété industrielle et le droit d'auteur ont encouragé un grand nombre d'experts à enseigner sur le sujet. Il en est de même du droit antitrust.

\section{La coopération internationale en vue de la mise au point de règles communes}

Ce tableau ne peut se terminer sans évoquer les efforts que se sont consentis les Etats depuis longtemps pour explorer les possibilités pour se mettre d'accord sur des règles communes. Il suffira de rappeler que, dès 1849, von Savigny énumère des traités signés par la Prusse avec des Etats voisins et indique ne pas en être mécontent, au contraire, et que, dans le premier volume du Journal du droit international privé et de la jurisprudence comparée (1874), que nous connaissons également sous le nom de son premier directeur, Clunet, P. S. Mancini a écrit son article célèbre sous le titre «De l'utilité de rendre obligatoire pour tous les Etats, sous la forme d'un ou plusieurs traités internationaux, un certain nombre de règles générales du droit international privé pour assurer la décision uniforme des conflits entre les différentes législations civiles et criminelles». Apparemment, l'idée de coopération entre les Etats dans notre domaine était dans l'air. Il n'est pas nécessaire en ce moment de nous attarder plus longtemps là-dessus.

En Amérique latine, les premiers résultats ont pu être enregistrés avant que, ici même, à La Haye, Tobias M. C. Asser n'eût réussi à faire convoquer par le Gouvernement des PaysBas la première conférence diplomatique, en 1893, avec l'objectif expressément formulé de mettre au point des conventions de droit international privé. Le plan était ambitieux, certes, mais, effectivement, cette conférence a été la première d'un grand nombre d'autres. Dès le début, ce n'était pas seulement Asser qui avait l'intention de continuer et, comme le rappelle le baron Nolde dans son cours de 1936, pendant la conférence de 1925 les délégués se sont mis d'accord pour poursuivre les travaux à l'avenir et pour décider que, par conséquent, cette conférence serait considérée comme la cinquième. Aussi n’y a-t-il pas lieu de s'étonner de ce que, pendant la septième conférence, celle de 1951, on ait créé une organisation internationale à part entière sous le nom de « Conférence de La Haye de droit international privé ». Aujourd'hui, il s'agit d'une organisation mondiale qui compte quarante-six Etats membres dispersés dans tous les continents du globe. 
Tous ceux et toutes celles qui participent, ou ont participé, aux travaux de la Conférence et éprouvent une certaine responsabilité pour leur bon déroulement n’hésiteront pas à affirmer qu'elle a largement bénéficié du centre de réflexion de haut niveau que constitue l'Académie. La Conférence et l'Académie sont un peu des sœurs jumelles. Nombreux sont les enseignants de l'Académie qui ont représenté leur pays en tant que délégués à la Conférence et, en grand nombre, les rapporteurs que la Conférence a élus pour les différentes conventions s'étaient ou se sont acquis depuis une bonne réputation à l'Académie (P. Lagarde, A. von Mehren, A. E. von Overbeck,G. Parra-Aranguren, D. W. $M$. Waters, $M^{\text {me }}$ Pérez Vera, et d'autres encore dans un passé plus éloigné). Maintes fois, les membres du Bureau permanent de la Conférence, ainsi que des délégués qui y sont devenus de vieux routiers, sont venus présenter à l'Académie les résultats des travaux de la Conférence assortis le plus souvent des idées auxquelles ont abouti leurs expériences et leurs réflexions. On lira avec intérêt le cours de M. H. van Hoogstraten, l'un des pères fondateurs de la nouvelle organisation et qui en a été le premier secrétaire général. Et on saura qu'Alfred von Overbeck comme Georges Droz, le successeur de M. H. van Hoogstraten, se sont vus chargés d'un enseignement à plusieurs reprises, dont un cours général. Il en est de même de A. Dyer, M. L. Pelichet et J. H. A. van Loon.

L’image de sœurs jumelles, utilisée pour caractériser les rapports entre l'Académie, d'une part, et la Conférence, d'autre part, ne manquera pas de rappeler le cours remarqué de Bruno Oppetit, qui avait pour thème « Le droit international privé, droit savant » ${ }^{21}$. Apparemment sans être en désaccord, il cite un auteur qui estime que

« la communauté savante, par l'échange d'arguments et la controverse, était seule à même, par-delà les frontières, de renouer avec le jus commune et de provoquer une unification internationale du droit par l'intégration dans les ordres nationaux de concepts et solutions communs ».

Et lui d'ajouter que: «le caractère savant du droit international privé ... est sans doute l'un des effets, mais aussi l'une des causes, de l'influence exercée par la doctrine en la matière ".

Les Conventions de La Haye, tout comme celles de l'Amérique latine d'ailleurs, qu'elles soient signées et ratifiées par un nombre relativement élevé d'Etats ou non, sont devenues l'objet d'analyses et de commentaires scientifiques. Cela se constate à la lecture des cours de l'Académie. Le texte des conventions ainsi que les Actes et documents mis au point par les soins du Bureau permanent de la Conférence sont des sources d'informations et d'inspiration qui sont très appréciées tant par les chercheurs que par les législateurs des différents Etats qui éprouvent le besoin de combler des lacunes dans leurs règles de droit international privé. 
Il faut se rendre compte que l'idée de faire des efforts pour mettre au point des conventions dans le domaine du droit international privé ne soulève pas un enthousiasme général. Au début de cette com-munication, Etienne Bartin a été cité pour souligner qu’il faut éviter la voie de la facilité et que les vrais résultats sont difficiles à at-teindre, au prix de recherches approfondies et d'efforts intellectuels extrêmes, étant donné le lien étroit entre le droit interne d'un pays et son droit international privé. Le grand auteur a courtoisement exprimé son pessimisme. Tout récemment, il a trouvé un écho dans les observations acerbes et très critiques présentées par Y. Lequette ${ }^{22}$. Ces propos, si décourageants qu'ils puissent paraître, n'en sont pas moins un avertissement à ne pas passer outre. Il faut avancer, ne serait-ce qu'avec la plus grande circonspection et, étant donné que le droit international privé est un droit savant, avec l'appui des savants les mieux qualifiés. Aussi les Etats membres seraient-ils avisés d'envoyer de telles personnes en tant que leurs délégués. C’est, en effet, ce qu'ont fait souvent, mais pas toujours, ces Etats. L'optimisme prudent dont a fait preuve Henri Batiffol, qui, lui-même, a été l'un des délégués de la France à plusieurs reprises, parmi tant d'autres experts illustres, servira toujours de point de repère pour orienter les travaux de la Conférence.

Terminons ainsi ce tableau des cours pour passer à la deuxième partie de cette communication.

\section{L'autonomie des parties à un contrat et les lois de police}

Il y a une tension certaine entre les lois de police, dont le moins qu'on puisse dire est qu'elles sont très impératives et, par conséquent, restreignent et veulent restreindre la liberté des hommes, tandis que l'autonomie des parties à un contrat exprime plutôt une liberté très grande, sinon excessive. L'idée de l'autonomie ne faut-il pas qu'elle s'accommode avec celle de l'Etat de droit ? ${ }^{23}$ Pendant longtemps, et avec des variations dans le temps, l'autonomie des parties à un contrat, à savoir le fait que, en droit international privé, elles peuvent librement désigner la loi qui régit leur contrat, n’a pas été un acquis indiscutable, et si, désormais, cette liberté fait partie de l'acquis, pour reprendre une expression bien connue du langage de la Communauté européenne, la discus-sion ne se termine pas là, et cela justement puisque cette liberté, dénommée également l'autonomie de la volonté, ne cesse de mon-trer des aspects nouveaux. Il n'en reste pas moins que le fait que, en droit international privé, la notion de loi de police se soit dégagée, bien qu'avec beaucoup de points qui restent encore à élucider, a probablement aidé certains à surmonter leurs hésitations pour souscrire au principe de l'autonomie des parties à un contrat international. Commençons donc par le phénomène des lois de police.

Aux fins de cette communication, il ne s'agit que de faire quelques explorations dans les seuls cours publiés de l'Académie. Il va de soi que, autrement, les sujets choisis, déjà considérables, seraient nettement trop vastes.

\section{Les lois de police}

Préliminairement, il convient de souligner que le phénomène des lois de police ne se cantonne pas dans le domaine des contrats internationaux, aussi important qu'il soit pour ces contrats. Une vaccination obligatoire pour de jeunes enfants sur tout le territoire d'un Etat, par exemple, pourrait être considérée comme une entorse à la loi qui, selon la règle de conflit, régit la responsabilité parentale. L’obligation s’imposera néanmoins. 
Essayons de préciser de quoi il s'agit. Nous pourrons prendre comme point de départ l'article 7 de la Convention de Rome du 19 juin 1980 relative à la loi applicable aux obligations contractuelles. L'article porte, dans la version française, le titre de «Lois de police ». Cette convention est entrée en vigueur pour douze Etats membres de la Communauté européenne, auxquels se joindront bientôt trois autres. Elle a attiré l'intérêt des experts dans beaucoup d'autres pays. Par exemple, on y trouve, non les termes, mais l'idée, dans quelques conventions mises au point au sein de la Conférence de La Haye ainsi qu'à l'article 13 de la Convention interaméricaine de Mexico du 17 mars 1994 relative à la loi applicable aux contrats internationaux ${ }^{24}$. Il en est de même de la codification suisse du droit international privé de 1989 dont l'article 18 se sert de l'expression «dispositions impératives », et de la codification italienne de 1995 dont l'article 17 porte comme titre «Norme di applicazione necessaria» (à la différence de la version italienne de l'article 7 de la Convention de Rome : «Norme imperative»).

Le point de départ de l'article 7 de la Convention de Rome nous permet d'en retracer la genèse. Il n’y a pas de doute que cet article consacre la reconnaissance d'un phénomène qui a été au centre des discussions depuis la parution, en 1958 à Paris, de l'ouvrage célèbre de Phocion Francescakis sur le renvoi. Cet auteur a fait remarquer que certaines règles de droit ne se plient pas au mécanisme de la règle de conflit traditionnelle du fait qu'elles ont leur manière à elles pour déterminer leur champ d'application. Fran-cescakis les a baptisées provisoirement "règles d'application immé-diate », pour exprimer qu'elles s'appliquent sans le biais d'une règle de conflit. Cette expression a fait le tour du monde avant que son auteur lui-même n'ait fait savoir qu'il lui préférait le terme " lois de police », bien connu depuis les premiers jours du Code Napo-léon. On sait que son article 3, première phrase, se lit comme suit : «Les lois de police et de sûreté obligent tous ceux qui habitent le territoire.» Jusqu'alors, le rôle de cette phrase pour le droit international privé avait été assez mal précisé, sinon ignoré. On retrouve cette disposition dans d'autres pays où on a suivi l'exemple du Code civil pour la mise au point de la législation de droit privé.

Depuis que cette discussion a été lancée, on a relu von Savigny, pour redécouvrir qu’il avait déjà attiré l'attention sur des lois d'un caractère très impératif et qui résistent à l'application sans entrave des règles de conflit. Cet auteur leur attribue un caractère exceptionnel et souligne que c'est surtout l'intention du législateur qui détermine ce caractère. Il explique qu'une telle loi peut s'appuyer sur des motifs d'ordre moral, et en donne l'exemple de l'exclusion de la polygamie, ou encore sur des motifs tenant au salut public (publica utilitas) pour les différencier selon leur caractère politique, policier ou économique.

La Cour permanente de Justice internationale a glissé dans l'arrêt de 1929 qu'elle a rendu dans l'affaire des emprunts serbes une phrase qui passa inaperçue dans le cours de Niboyet de $1932{ }^{25}$ mais qui, ultérieurement, a été relevée par Henri Batiffol ${ }^{26}$, et qui nous intéresse. Il souligne que l'idée dont il s'agit était pratiquement inconnue à l'époque. La phrase de l'arrêt se lit comme suit :

«Il y a ... cependant lieu de rappeler qu'il se peut que la loi qui pourrait être jugée, par la Cour, applicable aux obligations de l'espèce, soit, sur un territoire déterminé, tenue en échec par une loi nationale de ce territoire, loi d'ordre public et d'application inéluctable bien que le contrat ait été conclu sous le régime d’une loi étrangère. » 
Dès 1931, l'expert allemand Neumeyer a présenté des observations qu'on ne retrouve que dans un rapport que Julliot de la Morandière a mis au point pour un comité spécial de la Conférence de La Haye. Selon ce rapport, Neumeyer a évoqué des règles de police générale, ainsi que des règles de police sanitaire ou sociale ou économique. Malheureusement, ce rapport de Julliot de la Morandière, dont on parlera encore, n’a été publié qu'en 1951. C’est Van Hoogstraten qui fait état de ce passage dans son cours de $1967^{27}$.

En 1941 et en 1942, Wilhelm Wengler ${ }^{28}$ et Konrad Zweigert ${ }^{29}$ ont fait preuve de se rendre compte de ce phénomène, mais on ne s'en est souvenu que plus tard. En 1953, le professeur suédois Torsten Gihl a donné un cours sur les lois politiques et le droit international privé. Il se concentra surtout sur des mesures confiscatoires et laissa dans l'ombre les lois de police qui ont toute notre attention. F. A. Mann enseigne en 1971 sur les conflits de lois et le droit public, mais les lois de police sont laissées de côté. Il en est globalement de même du cours de 1997 sur les «Mandatory Rules in International Contracts : the Common Law Approach», de M. Trevor C. Hartley, qui ne situe guère la jurisprudence anglaise dans le cadre de l'article 7, cela malgré l'entrée en vigueur de la Convention de Rome. Cependant, M. Peter Nygh, Australien averti, consacre un chapitre de son cours de 1995 à notre sujet sous le titre de «Mandatory Rules» ${ }^{30}$.

Il ne faut pas être surpris de constater que, dans une première phase, ce sont surtout des auteurs continentaux qui se sont occupés des lois de police. A l'Académie, Rodolfo de Nova, Henri Batiffol, Yvon Loussouarn et Allan Philip ${ }^{31}$, et quelques autres encore, ne se sont pas bornés à rapporter ce qui s'est passé, mais y ont consacré des développements très pénétrants.

Il y a d'abord le problème de la qualification. Quelles sont les règles et les lois qui devraient être qualifiées de "lois de police » ? Faut-il différencier : y a-t-il d'autres lois également très impératives pour lesquelles le vocable loi de police est moins approprié ? Si on se déclare partisan d'une acception large, on risque un rétrécissement très net du domaine des règles de conflit de lois. Et cela plairait à ceux et celles qui sont séduits par les sirènes américaines, surtout celle de Brainerd Currie et des siens, dont la governmental interest analysis accuse une ressemblance frappante et souvent signalée avec l'idée directrice qui est derrière les lois de police, à savoir que, dans la société moderne, l'intervention de l'Etat dans les rapports de droit privé serait indispensable et qu'il faudrait réserver aux lois qui servent d'instruments à cette intervention un caractère impératif et la priorité, ou la préséance, par rapport aux règles de droit privé traditionnel lesquelles n’auraient désormais qu'un rôle résiduel ou subsidiaire à remplir. Il ne faut pas exclure qu'on va dé-couvrir un peu partout des lois de police, et notamment qu'on va qualifier comme telles toutes les lois qui ont pour objectif de protéger des faibles tels les enfants, les ouvriers, les femmes (certaines femmes, pas toutes, bien entendu !), les patients et les consommateurs, comme si le droit privé traditionnel ne pouvait être, et ne s'est pas avéré être, le gardien éprouvé de tels inté-rêts. Bref, le risque existe que le rôle attribué aux lois de police ait un effet perturbateur dans l'ordre déjà délicat du droit internatio-nal privé.

Henri Batiffol et Yvon Loussouarn n’ont pas hésité à exprimer leurs réserves devant l'essor du phénomène des lois de police. Il vaut la peine de citer le premier : 
«La difficulté principale est que nul n’a pu donner une définition digne de ce nom du domaine dans lequel le législateur disposerait ainsi sans égard à aucune règle de conflit de lois. M. Francescakis a risqué la formule des lois dont «l'observation est nécessaire à la sauvegarde de l'organisation politique, sociale et économique du pays » ... Une pareille formule peut englober toutes les lois, car on ne voit guère quel domaine resterait à des lois d'un autre caractère. Son auteur, interpellé sur ce point, a répondu que les autres lois seraient celles qui concernent les relations entre personnes privées. Mais comme l'Etat légifère sur ces relations précisément parce que des intérêts généraux, c'est-à-dire de caractère politique, social ou économique sont en jeu, on ne voit plus ce qui échappe aux lois de police.» ${ }^{32}$

Et Y. Loussouarn de dire :

«Dans les Etats modernes, on peut dire que toute loi tend pratiquement à garantir des intérêts économiques ou sociaux. Ce n'est pas un privilège réservé aux seules lois de police. En réalité il existe entre les lois de police et les autres lois une simple différence de degré, ce qui rend le clivage beaucoup plus difficile.» ${ }^{33}$

Il n’en reste pas moins que ces réserves, clairement exprimées par les grands auteurs, n’ont pu faire obstacle à l'adoption des dispositions énumérées qui pourtant sont d'une date ultérieure.

Et, une fois identifiée une loi de police, la question se pose de savoir comment il faut en déterminer le champ d'application. L'objectif d'une telle loi semble dicter ce champ d'application ${ }^{34}$, mais cette directive encore a souvent l'air d'être passablement floue et, de ce fait, d'entraîner une incertitude peu confortable, ce qui renforce les réserves citées.

Il s'y ajoute la question de savoir quel devrait être le domaine d'application d'une règle de conflit traditionnelle par rapport à des lois de police. Si, selon la règle de conflit, la loi d'un Etat étranger est applicable, il n'est pas évident pour tout le monde que cette dési-gnation englobe les lois de police d'un tel Etat. Certes, la réponse affirmative facilite les choses dans la mesure où on se dispense d'une ex-plication du fait que sont appliquées des lois étrangères qui ont, plus ou moins, le caractère de droit public. Mais, par souci de clarté, n’est-il pas préférable de se résigner aux conséquences de ce que, désormais, il y a une pluralité de méthodes et d'accepter que le domaine des lois de police et celui des conflits de lois constituent des domaines distincts ? Dans cette manière de voir, la question de savoir si une loi de police s'applique ne dépend aucunement d'une règle de conflit traditionnelle, normale, bilatérale, qu'il s'agisse d'une loi de police du for ou d'une loi de police d'un autre Etat. 
Quelques années plus tard, en 1977, c'est Pierre Lalive qui consacre un chapitre de son cours à notre sujet ${ }^{35}$. Il rappelle que, dans la littérature internationale, plusieurs expressions sont en vogue qui, toutes, traduisent plus ou moins la même idée. Tout en soulignant qu' «il serait ridicule de nier la réalité sociale que ces expressions incertaines cherchent à désigner ou à cerner», l'auteur en présente une analyse qui permet de mesurer les problèmes qui doivent encore être élucidés. Et, à leur tour, ses compatriotes Alfred E. von Overbeck ${ }^{36}$ et Frank Vischer ${ }^{37}$ s'étendent sur le phénomène dans leurs cours généraux respectifs, le dernier en faisant usage dans son cours en anglais de l'expression lancée en 1958 par Phocion Francescakis de «lois d'application immédiate ». Il reconnaît que le problème commence lorsqu'il s'agit d'identifier ces lois, tout en admettant qu'il n'est pas réaliste de confiner la catégorie aux règles constitutives de l'organisation étatique et celles qui assurent son fonctionnement. Par cette affirmation, il fait savoir qu'il considère la description de Francescakis comme trop étroite. Il se rend compte du danger que l'ensemble de ces lois va reléguer le système des conflits de lois au deuxième plan et fait remarquer que le texte de l'article 7 de la Convention de Rome ne contient aucun frein à cet égard, ce qui pourrait s'interpréter comme une invitation d'en faire largement usage. On pourrait voir se dessiner, et craindre, un « renouveau du particularisme », titre du cours qu'a professé Juan Antonio Carrillo Salcedo en 1978 et dont l'objet principal est notre thème d'au-jourd'hui.

Les réserves qu'on peut enregistrer augmentent encore quand il s'agit de savoir s'il est souhaitable d'admettre que des lois de police d'un Etat autre que celui du for puissent s’imposer. Ces réserves expliquent pourquoi les négociateurs de la Convention de Rome ont dû se résigner à la possibilité pour les Etats contractants d'exclure le premier alinéa de l'article 7 , malgré sa rédaction très modérée.

Le côté négatif des lois de police a attiré plus d'attention que le côté positif. Les lois de polices ne servent pas nécessairement des intérêts nationaux opposés à ceux d'autres Etats; elles peuvent aussi contribuer à promouvoir les intérêts de la communauté des Etats, par exemple la stabilité de l'ordre monétaire international et la protection des biens culturels.

On pourrait s'étendre longuement sur les lois de police. Elles constituent désormais, semble-t-il, un chapitre qui fait partie du noyau de la partie générale du droit international privé, ce d'autant qu'elles ont conquis leur place dans le droit positif. Tant des conventions multilatérales que des codifications récentes et bien préparées leur ont réservé des dispositions expresses, en les plaçant même dans leur partie centrale. Il n'en reste pas moins que la doctrine, qui ne peut ni ne veut ignorer leur existence, hésite à leur souhaiter la bienvenue, leur champ d'application étant toujours trop flou et leur préséance à l'égard du système des règles de conflit de lois étant potentiellement trop menaçante. Cependant, il faut bien admettre que la jurisprudence de quelques décennies est plutôt rassurante. Les lois de police n’ont pas envahi le droit international privé. La modération préconisée par la doctrine est devenue la règle, paraît-il.

Pour ce qui est de l'article 7 de la Convention de Rome, bientôt, espérons-le, la Cour de justice des Communautés européennes aura le pouvoir de surveiller son application.

Etant donné que la protection du bien commun dans le commerce juridique international a été renforcée grâce à l'instrument de la loi de police, il se peut qu'il soit permis de regarder l'autonomie des parties à un contrat international sous un autre jour. 
De nouveau, il n'est pas difficile de trouver un point de départ pour nos développements. En effet, l'autonomie de la volonté se trouve consacrée par deux conventions multilatérales et soigneusement préparées, à savoir la Convention de Rome de 1980 et la Convention de Mexico de 1994, toutes deux déjà citées à plusieurs reprises. Les parties à un contrat privé international ont la liberté de choisir la loi qui régira le contrat, non pas comme si cette loi ne remplissait que le rôle d'un ensemble de clauses, mais en tant que droit objectif, et ce avec l'effet que la loi choisie se substitue à la loi qui aurait été applicable à défaut de choix.

Il ne s'agit pas d'un principe de droit naturel, quoi que cela puisse signifier. Il ne s'agit pas non plus d'un principe qu'un Etat ne pourrait écarter que sous peine de violer le droit international public. L'autonomie n'existe que grâce au droit international privé des différents Etats. Il n'en reste pas moins qu'une unanimité impressionnante des Etats se dessine pour adhérer au principe de l'autonomie de la volonté, à tel point que, dans son cours de 1984, Ole Lando ${ }^{38}$ affirme que l'autonomie fait partie du noyau commun des systèmes juridiques, et, à son tour, en 1994, Andreas F. Lowenfeld estime même que, désormais, l'autonomie des parties est devenue une règle de droit coutumier ${ }^{39}$. Il s'agit d'un principe, ce qui réserve la possibilité d'exceptions, et notamment en faveur des parties faibles comme les travailleurs et les consommateurs ${ }^{40}$. Mais toujours est-il que le principe est là. Nous venons de réfléchir sur les lois de police. Il faut rappeler qu'elles restent intactes quelle que soit la portée de l'autonomie. Pour certains, cela est la condition implicite de leur adhésion à l'autonomie.

Quand on consulte les tomes du Recueil de l'Académie, on constatera qu'il n'en a pas toujours été ainsi pour ce qui concerne l'autonomie de la volonté.

Si l'essor de l'autonomie date de la fin du XIX ${ }^{\mathrm{e}}$ siècle, il y avait aussi des opposants redoutables. Aux Etats-Unis, Joseph Beale était le chef de file des antiautonomistes ${ }^{41}$. Cheshire paraît avoir été du même avis. A l'Académie, J.-P. Niboyet est monté sur l'estrade pour proclamer que, à son avis, l'idée de l'autonomie de la volonté, telle que nous la connaissons aujourd'hui, est une grosse erreur ${ }^{42}$ : «il n'y a pas d'autonomie de la volonté en matière de contrats, mais simplement une liberté des conventions », et cette proclamation n'a pas manqué d'avoir l'effet recherché. Après Niboyet, c'est Batiffol qui a exercé une influence certaine, bien au-delà de la frontière française, par sa doctrine selon laquelle la volonté des parties quant à la loi applicable à leur contrat n'est que l'un des facteurs qui entrent en ligne de compte quand il s'agit de « localiser » le rapport contractuel. Aux PaysBas, dans les années quarante, la doctrine était loin d'être unanime pour suivre Jan Kosters, partisan déclaré de l'autonomie, depuis la parution de son traité en 1917. Le désaccord ultérieur explique pourquoi le fameux projet Benelux, première version, de 1951, se montre très réticent, et cela conformément à l'avis de son auteur principal, E. M. Meijers.

Cependant, dans son cours de 1992, A. E. von Overbeck nous rappelle que, au sein de la Conférence de droit international privé, un mouvement a pris naissance en faveur de l'autonomie. En 1931, un comité s'est réuni pour préparer un projet de convention sur la vente. Le rapport de cette réunion a été mis au point par L. Julliot de la Morandière. A. E. von Overbeck qualifie ce rapport de texte remarquable. Il n’a été publié qu'en 1951, lors de la septième conférence diplomatique. L'auteur y plaide pour adopter l'autonomie sans restriction, à savoir pour choisir une loi, quelle qu'elle soit. Ce rapport est à la base de la Convention de La Haye de 1955 sur la vente, dont l'article 2, premier alinéa, est libellé comme suit : «La vente est régie par la loi interne du pays désigné par les parties contractantes. " Cette disposition revêt une plus grande importance qu'on ne le penserait pour le progrès de l'autonomie de la volonté. D’ailleurs, ce progrès n'était pas contrecarré par la jurisprudence de plusieurs pays. 
Toutefois, aux Pays-Bas, on a dû attendre l'arrêt célèbre de 1966 de l'affaire Alnati ${ }^{43}$ pour être entièrement rassuré. E. M. Meijers et d'autres, trouvant appui dans la version 1951 du projet Benelux, avaient mis en doute si un arrêt de 1946 faisait toujours autorité.

A. E. von Overbeck nous rappelle également que, pendant un certain temps, il y avait dans la doctrine non seulement un courant autonomiste à côté d'un courant antiautonomiste, mais un courant intermédiaire, actuellement un peu perdu dans l'oubli, et selon lequel la question de savoir si - et si oui dans quelle mesure - les parties à un contrat international ont la faculté de choisir la loi qui régira leur contrat doit trouver sa réponse dans le droit applicable en l'absence de choix par les parties. Adolf Schnitzer, auteur de la doctrine de la prestation caractéristique qu'il a exposée dans son cours de 1968 et qu'on retrouve dans la Convention de Rome de 1980, à l'article 4, lui, s'est montré partisan de ce courant intermédiaire, et avec lui d'autres encore. On trouve une trace de ce courant dans l'article 5, deuxième alinéa, de la Convention de La Haye de 1905 sur les effets du mariage :

« La même loi [à savoir la loi qui régit la validité et les effets du contrat de mariage] décide si et dans quelle mesure les époux ont la liberté de se référer à une autre loi ; lorsqu'ils s’y sont référés, c’est cette loi qui détermine les effets du contrat de mariage. "

Il est bien concevable que l'observateur peu familier avec le droit international privé reste perplexe devant cette curieuse autonomie de la volonté. La question se pose de sa légitimation. Une réponse bien connue revient à dire qu'il ne s'agit que d'une solution faute de mieux. Il faut que cette solution ait quand même quelque crédibilité en elle-même. Dans son cours général de 1986, dont l'idée directrice pour trouver soit la loi appropriée soit le for approprié est celle de la " proximité », Paul Lagarde fait apparaître qu'il trouve quelque mérite dans la thèse selon laquelle

« les différents droits nationaux seraient autant de cadres juridiques plus ou moins équivalents offerts aux contractants qui pourraient, librement, choisir de se soumettre à l'un d'entre eux»" ${ }^{44}$.

Cette thèse avait été soutenue par Pierre Gothot et, après lui, par Jean-Michel Jacquet. La même explication se retrouve dans le cours général, de 1991, de Georges Droz qui considère que:

«si toutes les lois européennes en matière de contrat ne sont pas nécessairement des plus modernes, elles reposent néanmoins sur des règles suffisamment savantes et suffisamment équitables pour qu'on soit sûr que le rapport juridique ne sera pas traité par elles d'une manière fantaisiste ou arbitraire» ${ }^{45}$.

L'autonomie des parties n'étant pas limitée à la loi des Etats membres de l'Union européenne, G. Droz devra étendre cette explication à la loi des autres Etats du monde. La règle qui permet de choisir la loi de l'Afghanistan ou celle de la Somalie pour un contrat qui ne se rattache aucunement à ces pays est-elle une bonne règle ? Quoi qu'il en soit, on ne trouve dans la jurisprudence aucun cas pour soutenir que la règle ne soit pas satisfaisante. Apparemment, dans la très grande majorité des situations, les parties à un contrat international et qui font usage de l'autonomie qui leur est gracieusement consentie ne commettent pas de bêtises ${ }^{46}$. Les parties elles-mêmes doivent être les arbitres de la sagesse de leur choix, comme l'a dit P. Nygh dans son cours de $1995^{47}$. Il ne semble pas qu'il soit imprudent, de la part des législateurs, des juges et des négociateurs de conventions de faire confiance aux parties. Il est plutôt préférable de couper court aux chicanes provenant de restrictions apportées à l'autonomie. 
Ce d'autant plus qu'il ne faut pas exclure qu'il y ait une tout autre raison à cela. Tout récemment, un praticien chevronné des affaires maritimes de Rotterdam a souligné que l'un des problèmes quotidiens auxquels il doit faire face c'est que, selon les règles de droit international privé qui s’appliquent à défaut de choix, la loi arriérée d'un pays lointain et connu pour son pavillon de complaisance devrait trouver application ${ }^{48}$. Cet auteur se plaint des complexités du droit international privé qui créeraient plutôt des obstacles à faire justice !... L'un des avantages de l'autonomie des parties pourrait être qu'elle permet précisément d'échapper à une telle loi arriérée et à remédier, ne serait-ce que partiellement, aux sérieux problèmes signalés.

Le moment est venu de terminer cette communication. On pourrait s'étendre encore longuement sur l'autonomie de la volonté. Notamment la question se pose de savoir si — et, si oui, à quelles conditions - l'autonomie de la volonté est ou devrait être admise dans d'autres domaines que celui des contrats.

\section{Conclusion}

Les richesses accumulées dans les tomes du Recueil des cours sont inestimables. J'ai pu le constater de nouveau en préparant cette communication.

On parle aujourd'hui de «globalisation », c'est-à-dire de l'extension et de l'intensification des activités économiques à une échelle mondiale. Si on se demandait quel a été, et quel est, le rôle de l'Académie, on pourrait dire qu'elle a apporté une immense contribution pour la "globalisation » des théories du droit international privé ainsi que des connaissances du droit positif.

En outre, il n'est pas exagéré de penser à une «synergie », dans la mesure où l'Académie a soutenu indirectement le travail des; législateurs, des juges et des négociateurs de conventions et queles développements du droit positif ont stimulé les réflexions des enseignants de l'Académie comme celles de leurs auditrices et auditeurs.

Pour l'avenir, trois points, parmi d'autres, doivent être retenus.

D'abord, il faudra veiller à éviter des lacunes dans les matières qui feront l'objet de l'enseignement.

Ensuite, il est difficile de ne pas penser que les richesses accumulées dans les tomes du Recueil ne portent pas toujours les fruits qu'elles méritent et que, par conséquent, l'accessibilité aux cours imprimés doit être un souci de première importance.

Un autre élément à prendre en considération est que, si les auditrices et les auditeurs ont des motifs valables pour s'en retourner satisfaits, ils seront les meilleurs ambassadeurs de « goodwill » possible de l’Académie.

L'Académie ne cesse d'avoir une grande mission à accomplir !

Et, pour terminer, je n’hésite pas à dire que je suis très fier de notre Académie ! 
1. J. H. van Roijen, " Holland and the Hague Academy of International Law » ; R.-J. Dupuy, «La contribution de l'Académie au développement du droit international». Les deux communications ont été publiées dans le Recueil des cours, tome 138 (1973), pp. 27-44 et pp. 45-74, respectivement.

2. E. A. Bartin, « La doctrine des qualifications et ses rapports avec le caractère national du conflit des lois », tome 31 (1931), pp. 561 ss., spéc. p. 619.

3. Henri Batiffol, «Principes de droit international privé », tome 97 (1959), pp. 431 ss. (442-443). Voir également E. Balogh, « Le rôle du droit comparé dans le droit international privé », tome 57 (1936), pp. 571 ss. (p. 596 : « un autre rôle du droit comparé, celui de pierre de touche des grandes théories de droit international privé »).

4. S. N. Lebedev, «International Commercial Arbitration in the Socialist Countries Members of the CMEA », tome 158 (1977), pp. 87-181.

5. M. M. Bogouslavski, « Doctrine et pratique soviétiques en droit international privé », tome 170 (1981), pp. 331-431.

6. C. Cardahi, « La conception et la pratique du droit international privé dans l'Islam. Etude juridique et historique », tome 60 (1937), pp. 507-650.

7. S. Mahmassani, « The Principles of International Law in the Light of Isla-mic Doctrine », tome 117 (1966), pp. 207-328 ; M. Charfi, «L'influence de la religion dans le droit international des pays musulmans », tome 203 (1987), pp. 321-454 ; J. Déprez, « Droit international privé et conflits de civilisations. Aspects méthodologiques. Les relations entre systèmes d'Europe occidentale et systèmes islamiques en matière de statut personnel ", tome 211 (1988), pp. 9-372.

8. K. R. R. Sastry, « Hinduism and International Law », tome 117 (1966), pp. 503-616.

9. K. N. Jayatilleke, « The Principles of International Law in Buddhist Doctrine », tome 120 (1967), pp. 441568.

10. Haopei Li, « Some Recent Developments in the Conflict of Laws of Succession ", tome 224 (1990), pp. 9-121.

11. Ph. Francescakis, «Problèmes de l’Afrique noire indépendante», tome 112 (1964), pp. 269-362; I. Szászy, «Le conflit de lois interpersonnel dans les pays en voie de développement», tome 138 (1973), pp. 81-202 ; U. U. Uche, "Conflict of Laws in a Multi-Ethnic Setting. Lessons from Anglophone Africa ", tome 228 (1991), pp. 273-438 ; A. K. Boye, « Le statut personnel dans le droit international privé des pays africains au sud du Sahara. Conceptions et solutions des conflits de lois. Le poids de la tradition négro-africaine personnaliste », tome 238 (1993), pp. 247-420 ; Alegría Borrás, « Les ordres plurilégislatifs dans le droit international privé actuel », tome 249 (1994), pp. 145-368, nos 37 ss., 96 ss.

12. A. Philip, « The Scandinavian Conventions on Private International Law », tome 96 (1959), pp. 241-348.

13. H. Valladão, «Le droit international privé des Etats américains », tome 81 (1952), pp. 1-116; id., «Conséquences de la différence de nationalité ou de domicile des époux sur les effets et la dissolution du mariage », tome 105 (1962), pp. 1-68; id., "Développement et intégration du droit international privé, notamment dans les rapports de famille », tome 133 (1971), pp. 413-528; Tatiana B. de Maekelt, « General Rules of Private International Law in the Americas. New Approach », tome 177 (1982), pp. 193-379 ; M. A. Vieira, "Le droit international privé dans le développement de l'intégration latino-américaine ", tome 130 (1970), pp. 351-454 ; id., « L'évolution récente de l’extradition dans le continent américain », tome 185 (1984), pp. 151-380; G. Parra-Aranguren, «Recent Develop-ments of Conflicts of Laws Conventions in Latin America », tome 164 (1979), pp. 55-170; id., "General Course of Private International Law: Selected Pro-blems », tome 210 (1988) ; A. Boggiano, «International Standard Contracts. A Comparative Study », tome 170 (1981), pp. 9-113; id., "The Contribution of the Hague Conference to the Development of Private International Law in Latin America. Universality and Genius Loci », tome 233 (1992), pp. 99-266.

14. F. K. Juenger, « General Course on Private International Law », tome 193 (1985).

15. F. K. Juenger, « General Course on Private International Law », tome 193 (1985), pp. 119-387 ; P. Hay, «Flexibility versus Predictability and Uniformity in Choice of Law. Reflections on Current European and United States Conflicts Law », tome 226 (1991), pp. 281-412.

16. P. A. Lalive, tome 155 (1977) ; François Rigaux, tome 213 (1989) ; Georges A. L. Droz, tome 229 (1991). II.

17. Henri Batiffol, « Les tendances doctrinales actuelles en droit international privé », tome 72 (1948), chap.

18. B. Goldman, «Les conflits de lois dans l’arbitrage international de droit privé », tome 109 (1963), pp. 347-486.

19. Karl Kreuzer, « La propriété mobilière en droit international privé », tome 259 (1996), pp. 9-317 ; Jean Voulgaris, « Le crédit-bail (leasing) et les institutions analogues en droit international privé », tome 259 (1996), pp. 319-412 ; D. W. M. Waters, « The Institution of the Trust in Civil and Common Law », tome 252 (1995), pp. 113-454. 
20. L. Galenskaya, «International Co-operation in Cultural Affairs », tome 198 (1986), pp. 265-322 ; L. V. Prott, " Problems of Private International Law for the Protection of the Cultural Heritage », tome 217 (1989), pp. 215-318 ; K. Siehr, « International Art Trade and the Law », tome 243 (1993), pp. 9-292.

21. Bruno Oppetit, « Le droit international privé, droit savant », tome 234 (1992), pp. 331-433, spéc. p. 375.

22. Yves Lequette, «Le droit international privé de la famille à l'épreuve des conventions internationales », tome 246 (1994), pp. 9-233.

23. Sur l'Etat de droit, Jacques-Yvan Morin, «L’Etat de droit: émergence d'un principe du droit international », tome 254 (1995).

24. ILM, vol. 33, 1994, p. 732.

25. J.-P. Niboyet, « Le rôle de la justice internationale en droit international privé : conflit des lois », tome 40 (1932), pp. 153-236.

26. Mélanges offerts à Charles Rousseau, Paris, 1974, p. 71, article repris dans le recueil Henri Batiffol, Choix d'articles rassemblés par ses amis, Paris, 1976, pp. 49 ss., spéc. p. 57.

27. M. H. van Hoogstraten, «La codification par traités en droit international privé dans le cadre de la Conférence de La Haye », tome 122 (1967), p. 409.

28. Wilhelm Wengler, "Die Anknüpfungen des zwingenden Schuldrechts im Internationalen Privatrecht », Zeitschrift für Rechtsvergleichung, 1941, pp. 168 ss.

29. Konrad Zweigert, « Nicht-erfüllung auf Grund ausländischer Leistungs-verbote », RabelsZ, 1942, pp. 283-307.

30. Peter E. Nygh, « The Reasonable Expectations of the Parties as a Guide to the Choice of Law in Contract and in Tort », tome 251 (1995), pp. 269-400.

31. A. Philip, "General Course on Private International Law », tome 160 (1978), pp. 1-73, chap. VII : «Public Law. »

32. H. Batiffol, « Le pluralisme des méthodes en droit international privé », tome 139 (1973), p. 138.

33. Y. Loussouarn, « Cours général de droit international privé », tome 139 (1978), p. 328.

34. Ph. Francescakis, «Quelques précisions sur les « lois d’application immédiate » et leurs rapports avec les règles de conflits de lois », Revue critique de droit international privé, 1966, pp. 1-18. (P. 14: "Les lois d'application immédiate doivent pouvoir dépendre de critères d'application conformes à leur but. »)

35. P. A. Lalive, « Tendances et méthodes en droit international privé », tome 155 (1977), pp. 120 ss.

36. A. E. von Overbeck, «Les questions générales du droit international privé à la lumière des codifications et projets récents », tome 176 (1982), pp. 9-258, spéc. pp. 177 ss.

37. F. Vischer, « General Course on Private International Law », tome 232 (1992), pp. 9-256, spéc. pp. 153174. Voir aussi Andreas Bucher, « L'ordre public et le but social des lois en droit international privé », tome 239 (1993), pp. 9-116.

38. O. Lando, «The Conflict of Laws of Contracts. General Principles »,tome 189 (1984), pp. 225-447, spéc. p. 237, avec l'accord de M. A. Boggiano, « The Contribution of the Hague Conference to the Development of Private Inter-na-tional Law in Latin America. Universality and Genius Loci », tome 233 (1992), p. 139.

39. A. F. Lowenfeld, «International Litigation and the Quest for Reason-a-bleness », tome 245 (1994), p. 256.

40. O. Lando, « The Conflict of Laws of Contracts. General Principles », tome 189 (1984), p. 302.

41. Bernard Audit, «Le caractère fonctionnel de la règle de conflit. (Sur la « crise » des conflits de lois) », tome 186 (1984), p. 293.

42. J.-P. Niboyet, « La théorie de l’autonomie de la volonté », tome 16 (1927), pp. 1-116, spéc. pp. 53-69.

43. Hoge Raad (cour de cassation), 13 mai 1966, Nederlandse Juris-pru-dentie, 1967, $\mathrm{n}^{0} 3$; Revue critique de droit international privé, 1967, p. 522, et la note.

44. Paul Lagarde, «Le principe de proximité dans le droit international privé contemporain », tome 196 (1986), $\mathrm{n}^{\circ} 50$.

45. Tome 229 (1991), p. 253.

46. Bernard Audit, «Le caractère fonctionnel de la règle de conflit. (Sur la « crise » des conflits de lois) », tome 186 (1984), p. 291.

47. Peter Nygh, "The Reasonable Expectations of the Parties as a Guide to the Choice of Law in Contract and in Tort », tome 251 (1995), p. 307.

48. H. Boonk, Zeerecht en IPR, Deventer, 1998. 